\title{
Atomic Tiles: Manipulative Resources for Exploring Bonding and Molecular Structure
}

\author{
Alan L. Kiste, Rebecca G. Hooper, Gregory E. Scott, and Seth D. Bush* \\ ${ }^{\dagger}$ Department of Chemistry and Biochemistry, California Polytechnic State University, San Luis Obispo, California 93407, United \\ States
}

Supporting Information

\begin{abstract}
A simple manipulative resource, Atomic Tiles, is described for scaffolding the learning of Lewis structures without using algorithmic, rule-based methods of drawing. Students use Atomic Tiles to (1) create models of bonding that lead to drawing Lewis structures, (2) use the structures they create to infer patterns required for rational structures and common organic functional groups, (3) translate between Lewis structures and molecular models, and (4) use molecular models to identify isomers.
\end{abstract}

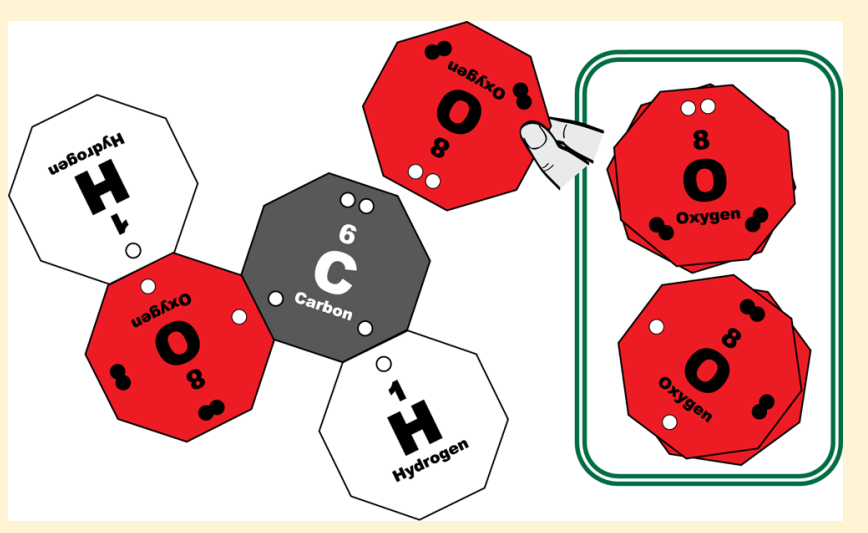

KEYWORDS: High School/Introductory Chemistry, First-Year Undergraduate/General, Organic Chemistry, Hands-On Learning/Manipulatives, Covalent Bonding, Lewis Structures, Molecular Properties/Structure

$\mathrm{M}$ any high school and college textbooks, and other published works, ${ }^{1-3}$ present the drawing of Lewis structures using algorithmic methods that suggest students (1) arrange atoms with the least electronegative atom as the central atom, and (2) distribute electrons to form bonds and lone pairs such that each atom has an octet of electrons. We believe that this algorithmic technique does not encourage students to learn many of the common patterns of bonding for closed-shell, uncharged main group atoms, and it may lead to the misconception that structures always have a single central atom. In addition, rule-based algorithms do not support thinking about common bonding patterns such as organic functional groups. These pattern-recognition skills are important for general chemistry, and for students continuing on to organic chemistry and beyond. ${ }^{4}$

One solution to the problem of having students memorize rules and algorithms that are only useful for drawing small, symmetrical structures is to encode the common bonding patterns for closed-shell, uncharged main group atoms within a simple manipulative. Manipulatives and games have been widely used to teach common polyatomic ions, periodicity, and many other concepts, ${ }^{5-7}$ and molecular models have been used to aid students in visualizing molecular shapes for over a century. ${ }^{8}$ Atomic Tiles were designed as a manipulative with affordances that scaffold correct Lewis structure building of highly complex molecules right from the start making it easy to extend the introductory topic of bonding beyond simple inorganic molecules. As students use the tiles, they can develop pattern-recognition skills to discern common bonding patterns as well as closed-shell formalisms for main group elements. As students' skills grow, additional Atomic Tiles can be added to introduce formal charge.

Drawing Lewis structures is not the end goal of this activity, but rather it is using Lewis structures to explore notions of molecular structure and isomerism, concepts that lead to polarity and properties such as melting point and boiling point later in our curriculum. Informed by research describing how students develop representational competence in chemistry, we have developed an activity using Atomic Tiles designed to provide students with opportunities to (1) create Lewis structures, (2) use the structures they create to infer patterns required for rational structures and common organic functional groups, (3) translate between Lewis structures and molecular models, and (4) use molecular models to identify isomers. ${ }^{9-13}$

\section{ATOMIC TILES}

Atomic Tiles are a flexible platform for introducing and reinforcing basic covalent bonding theory through guided inquiry. Atoms or ions are fashioned into octagonal tiles with valence electrons depicted as either open (depicted as white) or closed (depicted as black) circles. Open circles depict electrons that are shared to form bonds while closed circles depict unshared, lone pair electrons. For example, Figure 1A highlights 


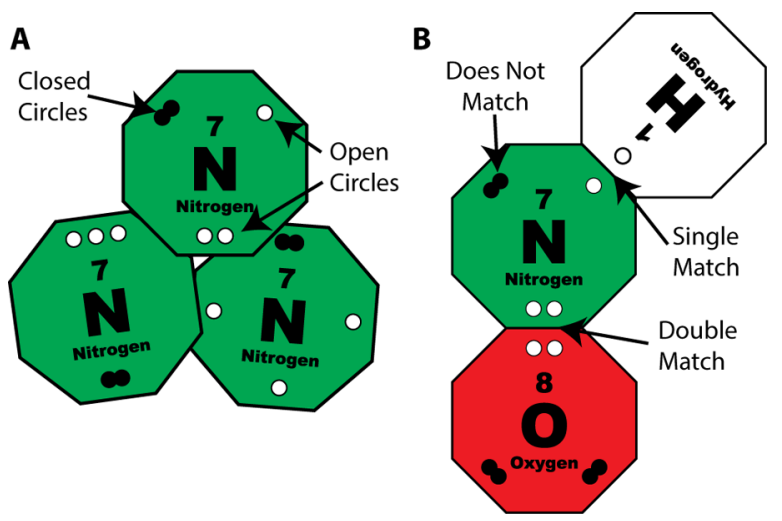

Figure 1. (A) Three configurations of a nitrogen tile. (B) Model of nitroxyl.

three configurations of a nitrogen Atomic Tile. Each variant has three open circles and two closed circles, depicting the nitrogen atom's five valence electrons. However, in each variant, the three open circles are distributed differently. Atomic Tiles can be combined to form bonds and ultimately molecules by matching open circles. Figure $1 \mathrm{~B}$ is an Atomic Tiles model of nitroxyl. In this model, the nitrogen atom has one single dot match (one single bond) with the hydrogen atom, one double dot match (one double bond) with the oxygen atom, and two closed circles that do not make matches (a lone pair). Atomic Tiles are a manipulative that makes physical the mental atomic puzzle pieces experienced chemists use to build molecular structures.

\section{IMPLEMENTATION}

We used this platform to develop a five-part activity for students in a college-level general chemistry course. In Part I, students explore valence electrons and bonding, with a focus on identifying bonding patterns. In Parts II and III, students build and translate their Atomic Tiles models into written Lewis dot structures. In Part IV, students explore isomers and basic functional groups. Finally, in Part V, students translate their two-dimensional models into three-demensional representations, ready to begin a discussion of intermolecular forces and structure and properties. This activity has been used with more than 3000 students in a college setting. A complete activity draft, instructor notes, and templates for Atomic Tiles are included in the Supporting Information.

Part I: Exploring Valence Electrons and Bonding (20 min) After Part I, students will be able to

- predict bonding behaviors of hydrogen, carbon, nitrogen, and oxygen using valence electrons;

- differentiate between bonding electrons and nonbonding electrons in Atomic Tiles models.

In part I, a two-person team is given a deck of 80 Atomic Tiles that contains a mixture of hydrogen, carbon, nitrogen, oxygen, fluorine, chlorine, sulfur, phosphorus, and silicon cards. Teams sort Atomic Tiles looking for similarities and differences among elements (Figure 2). First they sort their deck into four element piles: carbon, hydrogen, oxygen, and nitrogen (setting aside other elements for the time being). Then they sort these element tiles into each of their different configurations. Paying particular attention to the number of valence electrons and the number of possible bonds formed, teams address the following questions for each element.

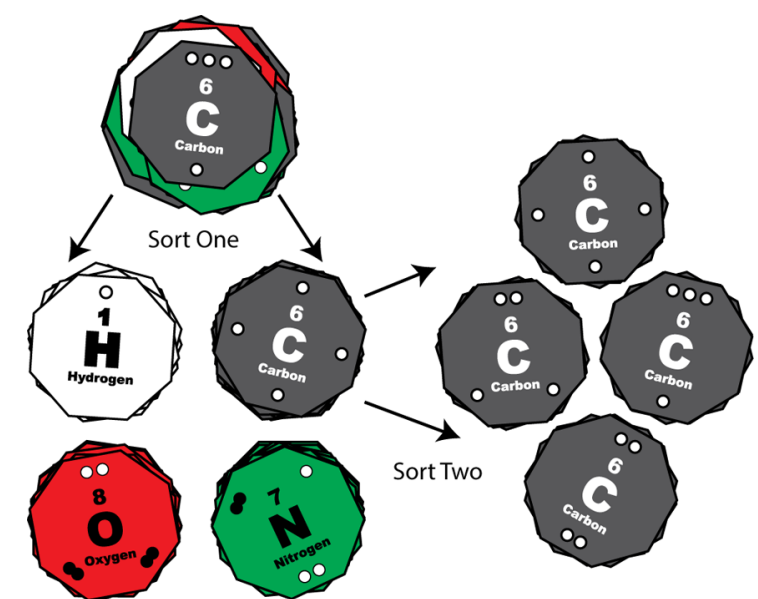

Figure 2. Card sorting: first sort by element, then sort by configuration.

(a) How many different configurations do you see?

(b) What is different about each configuration?

(c) What is the same about each configuration?

Part II: Making Molecules (30 min)

After part II, students will be able to

- build simple molecular models from chemical formulas using Atomic Tiles;

- recognize and explain bonding patterns for simple molecules;

- describe the octet rule.

In part II, teams use a deck of Atomic Tiles to build and explore a series of molecules from molecular formulas. From their models, they are asked to use two simple rules to build "good" molecules:

(1) The atoms in the molecule must match the molecular formula.

(2) All the open circles for each atom in the molecule must be matched.

Figure 3A highlights the decisions a student would have to make to build $\mathrm{H}_{2} \mathrm{O}$ using Atomic Tiles. First, using the
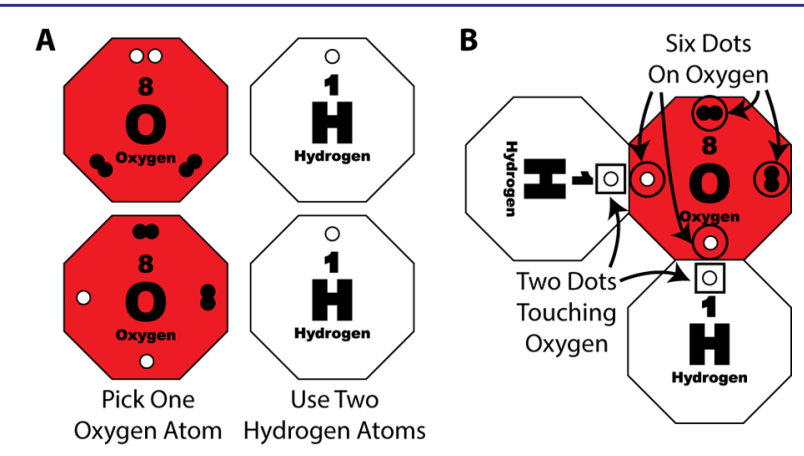

Figure 3. Molecule building: (A) tile choices for building water and (B) counting the total number of valence electrons around an atom after bonding.

molecular formula, students must recognize that their target molecule must have two hydrogen atoms and one oxygen atom. The students then begin to solve a puzzle. Which two hydrogen tiles and one oxygen tile can one pick such that they can match all the open circles? In this example the choice is obvious; there 
is only one configuration of hydrogen, and there are only two configurations of oxygen. However, this puzzling becomes more complex as molecular formulas get more complicated and there are more choices among element configurations to make.

Students are prompted to count the total number of valence electrons around each atom after bonding, looking for patterns for each molecule they build. Using the cards, the total number of valence electrons around an atom after bonding is the number of dots on the card of interest plus the number of dots touching the card of interest (Figure 3B). By examining a series of molecules, students "discover" the pattern that oxygen, nitrogen, and carbon have eight valence electrons associated with them after bonding, frequently stated as obeying the octet rule.

Part III: Lewis Dot Structures (30 min)

After part III, students will be able to

- make rational Lewis dot structures from chemical formulas using Atomic Tiles.

In part III, students translate Atomic Tile molecular models into written Lewis structures for several simple organic molecules. Again, students build Atomic Tiles models from the molecular formula and use these models to predict Lewis structures. In formaldehyde, for example (Figure 4), a single

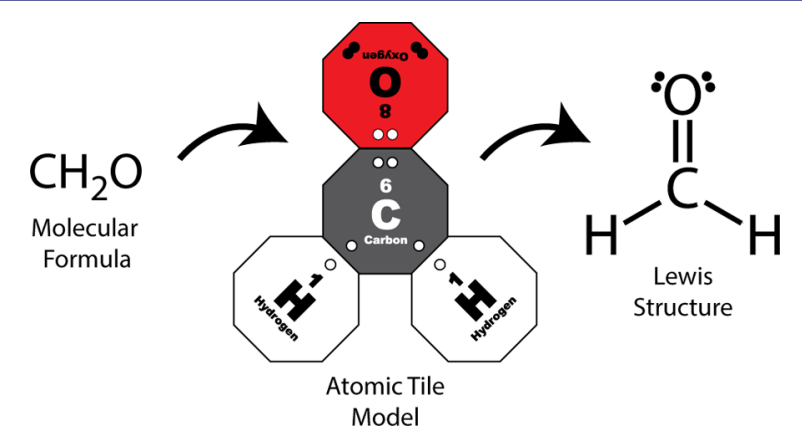

Figure 4. Building a Lewis structure for formaldehyde using Atomic Tiles.

dot match is replaced with a single line indicating a single bond, double dot matches become double bonds, and closed circles are depicted as two dots, or lone pairs. Had there been a triple dot match, it would have become a triple bond. By the end of this activity, most students move from using Atomic Tiles models to predict Lewis structures to using Atomic Tiles models to check written Lewis structures. Again, pattern recognition for carbon, nitrogen, oxygen, and hydrogen is emphasized.

Part IV: Isomers and Functional Groups (40 min)

After part IV, students will be able to

- create multiple rational Lewis dot structures of different isomers from chemical formulas;

- create rational Lewis dot structures with specific functional groups from chemical formulas.

Part IV extends students' understanding of Lewis structures to include structural isomers and simple carbon and oxygen functional groups. Three-dimensional molecular model kits are introduced for comparing potential structural isomers. Initially, students are given a molecular formula for simple molecules like $\mathrm{C}_{3} \mathrm{H}_{6} \mathrm{O}_{2}$ and asked to sketch as many structural isomers as they can and to identify all oxygen containing functional groups in each isomer. Figure 5 shows examples of two structural isomers with different functional groups that students might

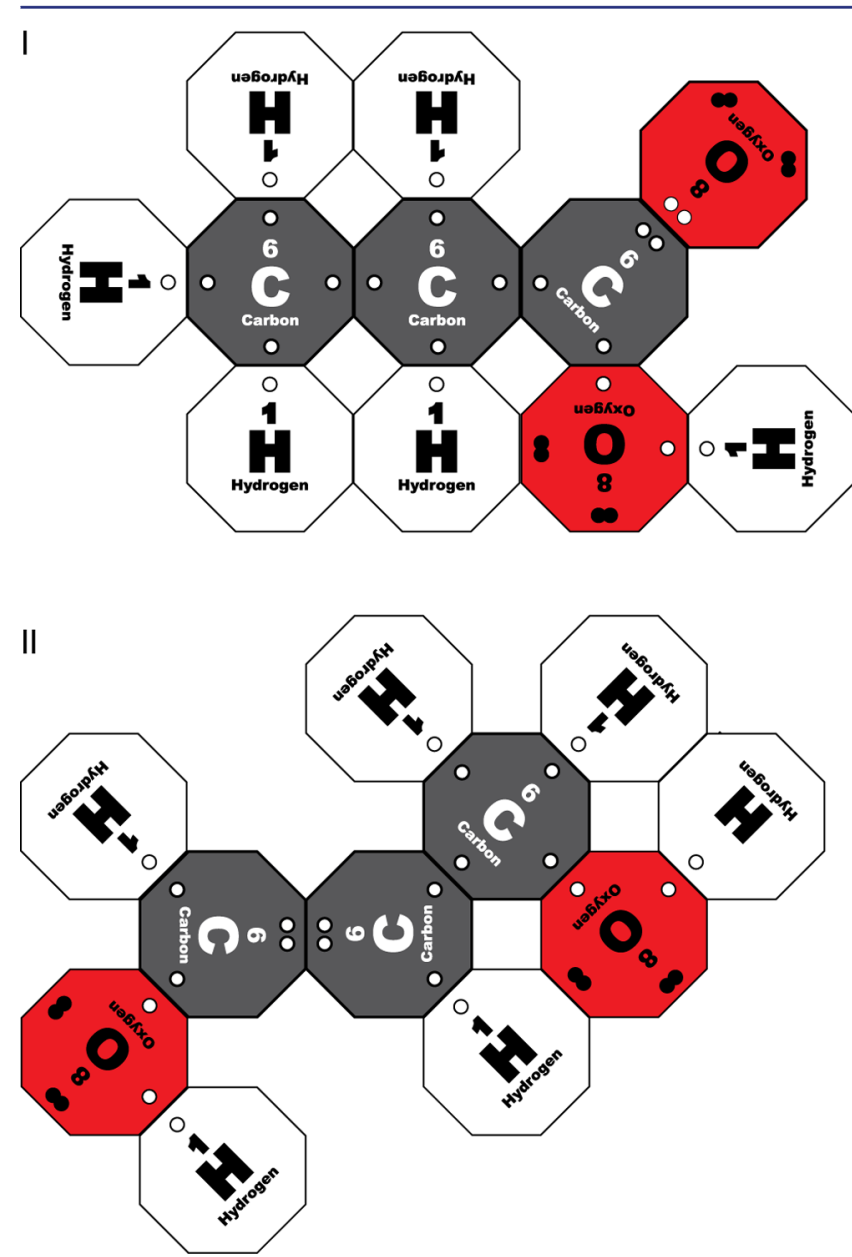

Figure 5. Two examples of structural isomers of $\mathrm{C}_{3} \mathrm{H}_{6} \mathrm{O}_{2}$ generated using Atomic Tiles.

create with Atomic Tiles for $\mathrm{C}_{3} \mathrm{H}_{6} \mathrm{O}_{2}$. While some students recognize that some isomers are more stable or more likely than others, at this stage we encourage students to be as creative as possible, following the basic rules embedded in their Atomic Tiles. Students are then given a simple molecular formula and asked to draw a Lewis structure with a specific functional group (e.g., carboxylic acid). Through exploring structural isomers and functional groups, students have an opportunity to refine the pattern-recognition skills developed in parts I-III while they learn new concepts.

Part V: VSEPR and 3D Shape (30 min)

After part V, students will be able to

- predict electronic and molecular geometry by identifying the number and type of electron groups around an atom;

- translate chemical formulas into three-dimensional molecular representations using Lewis structures and VSEPR.

In this culminating portion of this activity, students move from molecular formulas through Lewis structures toward predicting three-dimensional shapes using valence shell electron pair repulsion (VSEPR) theory. At this stage, most students are comfortable enough with the bonding patterns of organic molecules that they work independently or nearly 
independently of their Atomic Tiles decks. This experience lays the groundwork for our next unit on intermolecular forces.

\section{SUMMARY}

We have introduced a set of scaffolded activities using a simple manipulative resource that builds skills in drawing molecular structures based on pattern recognition. Anecdotally, we have included more questions with complex structures in our summative classroom assessments, in large part because the curriculum supported by this activity prepares our students to think about molecular structure in a more sophisticated way than generally supported by introductory chemistry textbooks. Students who have used the Atomic Tiles activity have performed relatively well on these assessment items. This activity can be further extended to examine formal charges and for exploring trends in the periodic table. Additional activities, including rules for a game, are available online. ${ }^{14,15}$

\section{AUTHOR INFORMATION}

\section{Corresponding Author}

*E-mail: sbush@calpoly.edu.

Notes

The authors declare the following competing financial interest(s): The patent for Atomic Tiles (US Patent number $7,955,083$ issued June 7 th, 2011) is owned by the Cal Poly Corporation on behalf of California Polytechnic State University. SDB is entitled to a fraction of the revenue generated by this patent should it ever prove commercially viable. To avoid a conflict of interest, a template for producing Atomic Tiles for non-commercial academic use has been included in the Supporting Information file.

\section{ACKNOWLEDGMENTS}

This work supported by Cal Poly Office of Research and Graduate programs and the Dean of the College of Science and Mathematics, Phil Bailey. The authors thank the numerous students and colleagues who have used these activities and provided helpful suggestions for improvement.

\section{REFERENCES}

(1) Miburo, B. B. Simplified Lewis Structure Drawing for Nonscience Majors. J. Chem. Educ. 1998, 75 (3), 317.

(2) Nassiff, P.; Czerwinski, W. A. Teaching Beginning Chemistry Students Simple Lewis Dot Structures. J. Chem. Educ. 2015, 92, 14091411.

(3) Silberberg, M. Chemistry: The Molecular Nature of Matter and Change; Mosby: St. Louis, MO, 1996.

(4) Cooper, M. M.; Grove, N.; Underwood, S. M.; Klymkowsky, M. W. Lost in Lewis Structures: An Investigation of Student Difficulties in Developing Representational Competence. J. Chem. Educ. 2010, 87 (8), 869-874.
(5) Antunes, M.; Pacheco, M. A. R.; Giovanela, M. Design and Implementation of an Educational Game for Teaching Chemistry in Higher Education. J. Chem. Educ. 2012, 89 (4), 517-521.

(6) Russell, J. V. Using Games to Teach Chemistry: An Annotated Bibliography. J. Chem. Educ. 1999, 76 (4), 481-484.

(7) Ruddick, K. R.; Parrill, A. L. JCE Classroom Activity \#113: An Interlocking Building Block Activity in Writing Formulas of Ionic Compounds. J. Chem. Educ. 2012, 89 (11), 1436-1438.

(8) McBride, J. M. Models and Structural Diagrams in the 1860 's. https: / / webspace.yale.edu/chem $125 / 125$ / history99/ 6Stereochemistry/models/models.html (accessed May 2016).

(9) Kozma, R.; Russell, J. Modelling Students Becoming Chemists: Developing Representational Competence. In Visualization in Science Education; Gilbert, J. K., Ed.; Springer: Dordrecht, 2005; pp 121-145.

(10) Kozma, R. Material and Social Affordances of Multiple Representations for Science Understanding. In Knowledge-in-theMaking: Epistemological Aspects of Using Inscriptions During Teaching and Learning Science. Symposium Conducted at the Annual Meeting of the American Educational Research Association (AERA); Toth, E., Ed.; Seattle, WA, 2001.

(11) Schank, P.; Kozma, R. Learning Chemistry through the Use of a Representation-Based Knowledge Building Environment. J. Comput. Math. Sci. Teach. 2002, 21 (3), 253-279.

(12) Kozma, R.; Chin, E.; Russell, J.; Marx, N. The Roles of Representations and Tools in the Chemistry Laboratory and Their Implications for Chemistry Learning. J. Learn. Sci. 2000, 9 (2), 105143.

(13) Stieff, M.; Bateman, Jr., R. C.; Uttal, D. H. Teaching and Learning with Three-Dimensional Representations. In Visualization in Science Education; Gilbert, J. K., Ed.; Springer: Dordrecht, 2005.

(14) Atomic Tiles Tile Sets. http://www.atomic-tiles.com/tilesets. html (accessed Jul 2016).

(15) Atomic Tiles: An Exploration of Atoms and Molecules http:// www.venturaes.com/iosapps/atomic_tiles.html (accessed Jul 2016). 